\section{Management of atrial fibrillation in congestive heart failure patients}

Patients with congestive heart failure often suffer from atrial fibrillation. As each condition exacerbates the other, this poses problems in terms of management. A new study by Hsu et al. has investigated the use of curative catheter ablation for atrial fibrillation in heart failure patients, in order to determine how the treatment affects left-ventricular function.

This prospective study included 58 patients with congestive heart failure and 58 matched controls, all of whom underwent catheter ablation to treat drug-resistant atrial fibrillation. During the mean follow-up of $12 \pm 7$ months, sinus rhythm was maintained in a similar proportion of heart failure and control patients. In most cases, sinus rhythm was achieved without the use of antiarrhythmic drugs. Leftventricular function improved significantly $(P<0.001)$ in the congestive heart failure patients, in terms of ejection fraction and fractional shortening. Left-ventricular dimensions, exercise capacity, symptoms and quality of life also improved significantly in this group. These improvements corresponded to a statistically significant improvement in the mean NYHA class, from $2.3 \pm 0.5$ before ablation to $1.4 \pm 0.5$ 1 month later; this improvement was maintained at 12 months.

Patients with concurrent structural heart disease and adequate rate control before ablation also benefited from the therapy. These results suggest that atrial fibrillation has an important negative impact on heart function, which can reverse after catheter ablation and maintenance of sinus rhythm without antiarrhythmic drugs.

Original article Hsu L-F et al. (2004) Catheter ablation for atrial fibrillation in congestive heart failure. $N$ Engl J Med 351: 2373-2383

\section{Prophylactic ICD therapy after MI: no survival benefit}

The risk of ventricular arrhythmias in patients with heart disease can be reduced using implantable cardioverter defibrillator (ICD) therapy. Whether prophylactic ICD use is beneficial in patients who have recently suffered a myocardial infarction is, however, unknown. The international Defibrillator in Acute Myocardial Infarction Trial was designed to answer this question.

The investigators enrolled patients who had had a myocardial infarction within the previous 6 to 40 days and who had severe leftventricular dysfunction and impaired cardiac autonomic function. They then compared mortality from any cause or from arrhythmia in patients who subsequently underwent prophylactic ICD therapy $(n=332)$ and those who did not $(n=342)$.

During the mean follow-up of $30 \pm 13$ months, there were significantly fewer deaths due to arrhythmia in the ICD group than in the control group. This benefit was masked, however, by the higher rate of death from nonarrhythmic causes in the ICD group. These 'nonarrhythmic' deaths were mostly from other cardiovascular causes. Mortality from any cause, therefore, was similar in the ICD and control groups.

Authors Hohnloser et al. conclude that prophylactic ICD therapy did not reduce overall mortality in this study, despite a significant reduction in the risk of death from arrhythmia.

Original article Hohnloser SH et al. (2004) Prophylactic use of an implantable cardioverter-defibrillator after acute myocardial infarction. N Engl J Med 351: 2481-2488

\section{Drug-eluting stents for small vessel revascularization}

Revascularization of small coronary arteries is problematic, not least because of the high rate of restenosis following stent implantation. A recent Italian trial has shown that sirolimuseluting stents reduce restenosis in small vessels compared with uncoated stents of the same design.

This multicenter, single-blind study included patients with ischemic heart disease who had a single, untreated atherosclerotic lesion in a small (less than or equal to $2.75 \mathrm{~mm}$ diameter) native coronary artery. Patients were randomized to percutaneous coronary revascularization using a sirolimus-eluting stent $(n=129)$ or an equivalent, uncoated stent $(n=128)$ that was angiographically indistinguishable.

Eight months after the procedure, the restenosis rate was significantly lower in 